\title{
Compositionality Results for Cardiac Cell Dynamics
}

\author{
Md. Ariful Islam ${ }^{1}$, Abhishek Murthy ${ }^{1}$, Ezio Bartocci ${ }^{2}$, \\ Antoine Girard ${ }^{3}$, Scott A. Smolka ${ }^{1}$, and Radu Grosu ${ }^{1,2}$ \\ 1 Department of Computer Science, Stony Brook University \\ 2 Department of Computer Engineering, Vienna University of Technology \\ ${ }^{3}$ Université Joseph Fourier, Grenoble, France
}

\begin{abstract}
We show that the 13-state sodium channel component of the Iyer et al. cardiac cell model can be replaced with a previously identified $\delta$-bisimilar 2-state Hodgkin Huxley-type abstraction by appealing to a small gain theorem. To prove this feedback compositionality result, we construct quadratic-polynomial exponentially decaying bisimulation functions between the two sodium channel models and also for the rest of a simplified version of the Iyer et al. model using the SOSTOOLS toolbox. Our experimental results validate the analytical ones. To the best of our knowledge, this is the first application of $\delta$-bisimilar, feedbackassisting, compositional reasoning in biological systems.
\end{abstract}

The Iyer et al. model (IMW) [3] is a physiologically detailed cardiac myocyte (ventricular) model that can be used to to simulate the change in a cell's transmembrane potential in response to an external electrical stimulus, also known as the Action Potential (AP). In this work, we ask "assuming that the $A P$ is

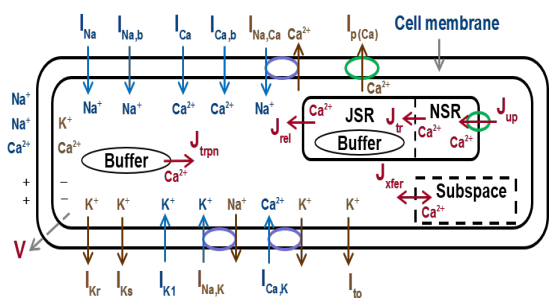

(a)

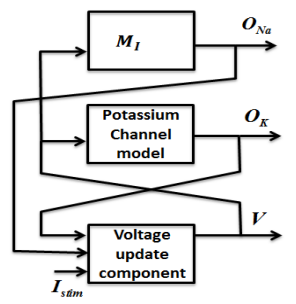

(b)

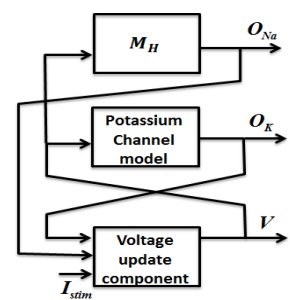

Fig. 1. (a)The IMW model, showing various currents. (b)The sodium channel components $M_{I}$ (detailed) and $M_{H}$ (abstract) composed with the potassium and voltage components forming the rest of a simplified version (IMW') of the IMW model.

the only observable, can we replace the sodium current component, $M_{I}$, of IMW with an equivalent model-order reduced Hodgkin Huxley (HH)-type model $M_{H}$ ?" The HH model [2], uses two variables $m$ and $h$ to model a squid neuron's transmembrane sodium current. In [4], we proposed an algorithm to identify $M_{H}$ that is $\delta$-bisimilar (equivalent) to the 13-state voltage-controlled $M_{I}$.

Compositionality of the equivalent sodium channels with the rest of the simplified IMW model (IMW-RT') can be established using Bisimulation Functions $(\mathrm{BFs})$ and a small gain theorem based on them. 
Definition 1. Consider two dynamical systems $\Sigma_{i}$, as per [1], but with $g_{i}$ : $\mathcal{X}_{i} \rightarrow \mathcal{Y}_{i}$, being the output functions that map a state to $\mathbf{y}_{i} \in \mathcal{Y}_{i} \subseteq \mathbb{R}^{p}$. Let $R_{\delta}=\left\{\left(\mathbf{x}_{1}, \mathbf{x}_{2}\right) \mid\left\|g_{1}\left(\mathbf{x}_{1}\right)-g_{2}\left(\mathbf{x}_{2}\right)\right\| \leq \delta\right\}$.A smooth function $S: R_{\delta} \rightarrow \mathbb{R}_{0}^{+}$is a $\delta$-Restricted BF ( $\delta$-RBF) over $\Sigma_{1}$ and $\Sigma_{2}$ if:

$$
\left\|g_{1}\left(\mathbf{x}_{1}\right)-g_{2}\left(\mathbf{x}_{2}\right)\right\| \leq S\left(\mathbf{x}_{1}, \mathbf{x}_{2}\right)
$$

and there exists $\lambda>0, \gamma \geq 0$ such that $\forall \mathbf{u}_{1} \in \mathcal{U}_{1}, \mathbf{u}_{2} \in \mathcal{U}_{2}$,

$$
\frac{\partial S}{\partial \mathbf{x}_{1}} f_{1}\left(\mathbf{x}_{1}, \mathbf{u}_{1}\right)+\frac{\partial S}{\partial \mathbf{x}_{2}} f_{2}\left(\mathbf{x}_{2}, \mathbf{u}_{2}\right) \leq-\lambda S\left(\mathbf{x}_{1}, \mathbf{x}_{2}\right)+\gamma\left\|\mathbf{u}_{1}-\mathbf{u}_{2}\right\|
$$

Theorem 1. Let $\Sigma_{1}, \Sigma_{2}$ and $\Sigma_{3}$ be three dynamical systems. Let $\Sigma_{13}$ and $\Sigma_{23}$ be interconnections (as defined in [1]) of $\Sigma_{3}$ with $\Sigma_{1}$ and $\Sigma_{2}$ respectively. Let $S_{12}$ be a $\delta$-RBF between $\Sigma_{1}$ and $\Sigma_{2}$ and $S_{3}$ be $\delta$-RBF for $\Sigma_{3}$. We denote by $\lambda_{12}$ and $\gamma_{12}\left(\lambda_{3}\right.$ and $\gamma_{3}$ respectively) the constants such that Eq. (2) holds. If $\frac{\gamma_{12} \gamma_{3}}{\lambda_{12} \lambda}<$ 1 , then there exists a BF $S$ between $\Sigma_{13}$ and $\Sigma_{23}$ of the form $S\left(\mathbf{x}_{13}, \mathbf{x}_{23}\right)=$ $\alpha_{1} S_{12}\left(\mathbf{x}_{1}, \mathbf{x}_{2}\right)+\alpha_{2} S_{3}\left(\mathbf{x}_{3}, \mathbf{x}_{3}^{\prime}\right)$ where, $\mathbf{x}_{13}=\left[\mathbf{x}_{1}, \mathbf{x}_{3}\right]$, and $\mathbf{x}_{23}=\left[\mathbf{x}_{2}, \mathbf{x}_{3}^{\prime}\right]$ The real constants $\alpha_{1}$ and $\alpha_{2}$ can be chosen as in Eq.4 of [1] by replacing $\lambda_{1}=\lambda_{12}$, $\gamma_{1}=\gamma_{12}, \lambda_{2}=\lambda_{3}$ and $\gamma_{2}=\lambda_{3}$.

The two BFs, 1) between $M_{I}$ and $M_{H}$ and 2) for IMW-RT' were identified in the SOSTOOLS toolbox [5] by adding the following constraint along with the ones that define a BF: $S\left(\mathbf{x}, \mathbf{x}^{\prime}\right)-\left\|g_{1}(\mathbf{x})-g_{2}\left(\mathbf{x}^{\prime}\right)\right\| \leq \delta$. The parameter $\lambda$ was fixed to either $10^{-4}$ and $10^{-5}$ for the two BFs and $\gamma$ was found to be $10^{-6}$, which resulted in the small-gain condition being satisfied. Fig. 2 shows experimental evidence of the model equivalence on replacing $M_{I}$ by $M_{H}$.

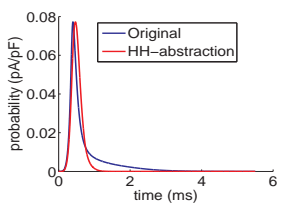

(a) Na channel conductance.

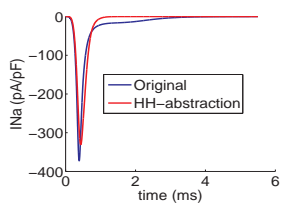

(b) $I_{N a}$ current.

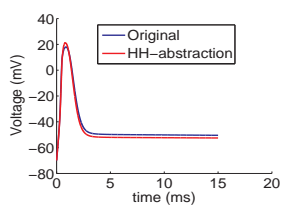

(c) APs.

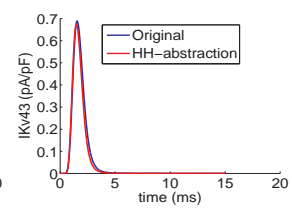

(d) $I_{K v 43}$ current.

Fig. 2. IMW' was stimulated using $-100 \mathrm{pA} / \mathrm{pF}$ stimulus with $M_{I}$ and then $M_{H}$. The resulting mean L1 errors were $O_{N a}: 9.15 \times 10^{-4}, I_{N a}: 3.8 p A / p F, I_{K v 43}: 0.0078 p A / p F, V: 2.29 m V$.

\section{References}

1. A. Girard. A composition theorem for bisimulation functions. Pre-print, 2007. arXiv:1304.5153.

2. A. L. Hodgkin and A. F. Huxley. A quantitative description of membrane current and its application to conduction and excitation in nerve. Journal of Physiology, 117:500-544, 1952.

3. V. Iyer, R. Mazhari, and R. L. Winslow. A computational model of the human left-ventricular epicardial myocytes. Biophysical Journal, 87(3):1507-1525, 2004.

4. A. Murthy, M. A. Islam, E. Bartocci, E. Cherry, F. H. Fenton, J. Glimm, S. A. Smolka, and R. Grosu. Approximate bisimulations for sodium channel dynamics. In Proc. of CMSB'12, LNCS. Springer, 2012.

5. S. Prajna, A. Papachristodoulou, P. Seiler, and P. A. Parrilo. SOSTOOLS: Sum of squares optimization toolbox for MATLAB, 2004. 